\title{
LES SOURCES INTÉGRÉES de photons intriqués au coeur des technologies quantiques
}

\author{
Maria AMANTI \\ Florent BABOUX \\ Sara DUCCI \\ Laboratoire Matériaux et \\ Phénomènes Quantiques, \\ Université Paris Diderot, \\ Sorbonne Paris Cité, \\ CNRS-UMR 7162, France \\ sara.ducci@univ-paris-diderot.fr
}

\section{Du paradoxe EPR aux technologies photoniques quantiques}

Le concept d'intrication, terme inventé par Schrödinger en 1935, fut au centre d'un célèbre article publié la même année par Einstein, Podolsky et Rosen [1]. Dans cet article, les auteurs analysèrent les prédictions des mesures des corrélations pour un état de deux particules pour lesquelles les propriétés d'ensemble sont définies, mais pas les propriétés individuelles. Ils firent deux hypothèses : la première, appelée " réalisme » est que "si, sans perturber un système, on peut prédire avec certitude la valeur d'une grandeur physique, alors il existe un élément de réalité correspondant à cette quantitéphysique ». La deuxième hypothèse, appelée localité, est que la mesure effectuée sur une particule ne peut pas influencer les propriétés de l'autre quand les particules n'interagissent pas entre elles. À partir de ces deux hypothèses, les auteurs argumentèrent que la description de la réalité donnée par les lois de la mécanique quantique n'était pas complète. Cet argument souleva des débats acharnés parmi les fondateurs de cette théorie et devint testable expérimentalement grâce au travail de Bell en 1964, qui établit des inégalités montrant que, pour certaines combinaisons des paramètres de mesure, la mécanique quantique prédit des corrélations qui
L'intrication est un des aspects les plus fascinants et contre-intuitifs des systèmes quantiques, devenue une ressource clé dans tous les domaines de l'information quantique, des communications à la métrologie, des simulations au calcul. La photonique quantique intégrée est en train de jouer un rôle moteur pour le développement de sources de photons intriqués de plus en plus performantes et leur insertion dans des circuits quantiques complexes. sont incompatibles avec les hypothèses de réalisme et localité.

À partir de la fin des années 70, plusieurs générations de tests expérimentaux des inégalités de Bell ont démontré de façon convaincante que l'intrication existe et que la nature ne peut pas être décrite par une théorie locale réaliste au sens d'Einstein, Podolsky et Rosen. L'intérêt pour les expériences sur l'intrication est toujours très fort pour l'exploration des limites entre la physique classique et quantique et l'extension des concepts de non-localité à des régimes relativistes. Plusieurs groupes à travers le monde, en particulier en Chine, sont ainsi impliquées dans une sorte de « course spatiale quantique » qui consiste à envoyer dans l'espace des satellites équipés avec des technologies quantiques pour tester la physique quantique dans de nouveaux régimes.

En plus de ces motivations fondamentales, ces 30 dernières années ont vu l'explosion du domaine de l'information quantique, dont l'objectif est d'implémenter de nouvelles formes de communication, calcul, simulation et mesure basées sur l'utilisation de systèmes quantiques, et où l'intrication joue un rôle central. En particulier, les états quantiques photoniques présentent un avantage particulier par rapport aux autres systèmes pour la
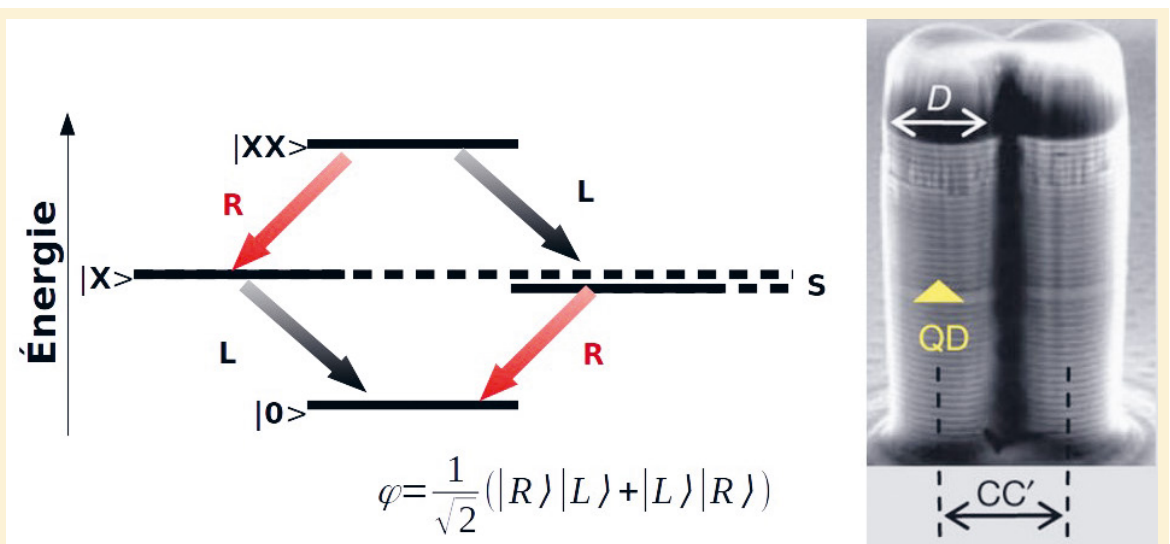

Figure 1. Image au microscope électronique à balayage d'une «molécule photonique » contenant une boitte quantique dans un de ses deux piliers. Ce dispositif émet des photons intriqués en polarisation par cascade radiative biexcitonique. Deux chemins de recombinaison radiative sont possibles à travers deux états excitoniques. Si la séparation en énergie $\mathrm{S}$ entre ces deux états est nulle, les deux chemins sont indiscernables et les deux photons émis dans la cascade sont intriqués en polarisation. (crédits image P. Senellart, Centre de Nanosciences et Nanotechnologies) 


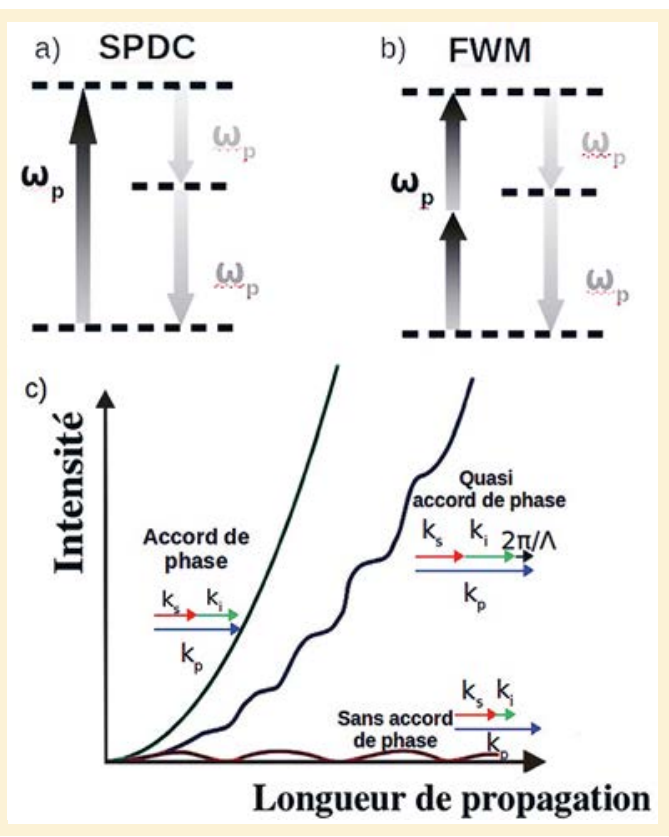

Figure 2. (a, b) Schémas des processus de conversion non-linéaire utilisés pour produire des paires de photons (SPDC : spontaneous parametric downconversion ; FWM : four-wave mixing). Dans le mélange à trois (quatre) ondes, un (deux) photon(s) de pompe génère(nt) une paire de photons appelés signal et complémentaire. Dans ce processus l'énergie est conservée et, pour avoir une conversion efficace, les ondes doivent se propager en phase (condition d'accord de phase). (c) Puissance générée en fonction de la longueur de propagation pour diverses conditions d'accord de phase. transmission d'information, car les photons se comportent naturellement comme des qubits « volants » se déplaçant à grande vitesse sur des longues distances, tout en étant presque immunes à la décohérence. Des photons intriqués ont ainsi déjà été transmis à des distances record de $300 \mathrm{~km}$ en utilisant des fibres optiques et plus récemment 1200 km grâce à l'utilisation du satellite spatial chinois Micius. Mais l'utilisation de photons intriqués est aussi en train de faire progresser d'autres domaines de l'information quantique, comme la métrologie ou la simulation. Pour que ces nouvelles technologies puissent fonctionner et se déployer à grande échelle, il est nécessaire de développer des composants quantiques pratiques, fiables et adaptés à une production de masse.

Dans ce contexte, la photonique quantique intégrée suscite actuellement un intérêt croissant. Elle permet en effet de réaliser à la fois des sources de photons intriqués efficaces, et de les combiner avec des circuits photoniques ayant une stabilité de phase intrinsèque et permettant donc d'implémenter des opérations complexes. En particulier les avancées remarquables sur les sources de photons intriqués basées sur des plateformes semiconductrices ouvrent la voie vers la démonstration de systèmes massivement parallèles pour la génération, la manipulation et la détection d'états quantiques de lumière sur puce [2].

\section{Processus physiques générant lintrication}

L'intrication peut être vue comme la généralisation à plusieurs particules du principe de superposition : l'état décrivant le système ne peut pas être factorisé, c'est-à-dire écrit comme un produit tensoriel de l'état de chaque particule. Pour produire des paires de photons intriqués, on doit utiliser un processus physique qui permette de les produire de deux façons différentes mais indiscernables. Cette condition peut être remplie soit au sein de la source elle-même, soit par manipulation de l'état généré par la

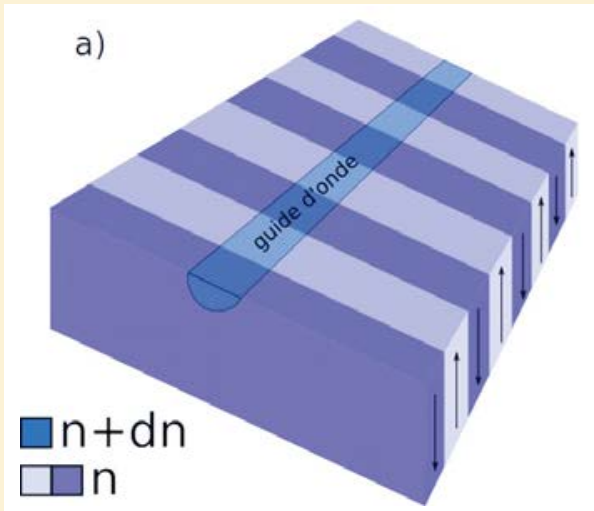

b)

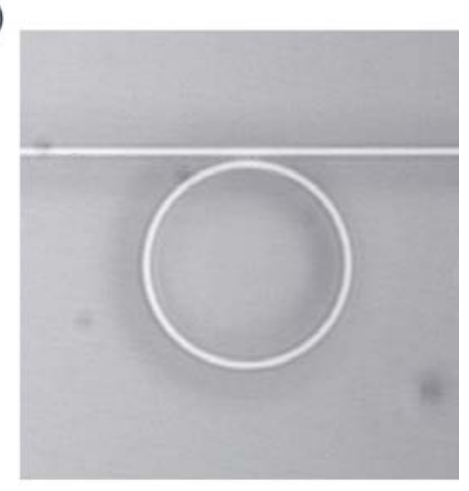

Figure 3. (a) Schéma d'un guide d'onde en niobate de lithium périodiquement polarisé (PPLN); la non-linéarité du matériau est inversée périodiquement et de façon permanente grâce à l'application d'un fort champ électrique à travers un réseau d'électrodes; la zone guidante à fort indice peut être créée en utilisant diverses techniques (crédit image O. Alibart, INPHYNI, UCA et CNRS).

(b) Image au microscope optique d'un micro anneau en silicium fabriqué sur la plateforme SOI. Les paires de photons sont générées par mélange à quatre ondes et émises dans les modes de résonance de l'anneau. Un nanofil couplé de façon évanescente à l'anneau permet de coupler le laser de pompe et collecter les paires de photons (crédit image D. Bajoni, Université de Pavia). 


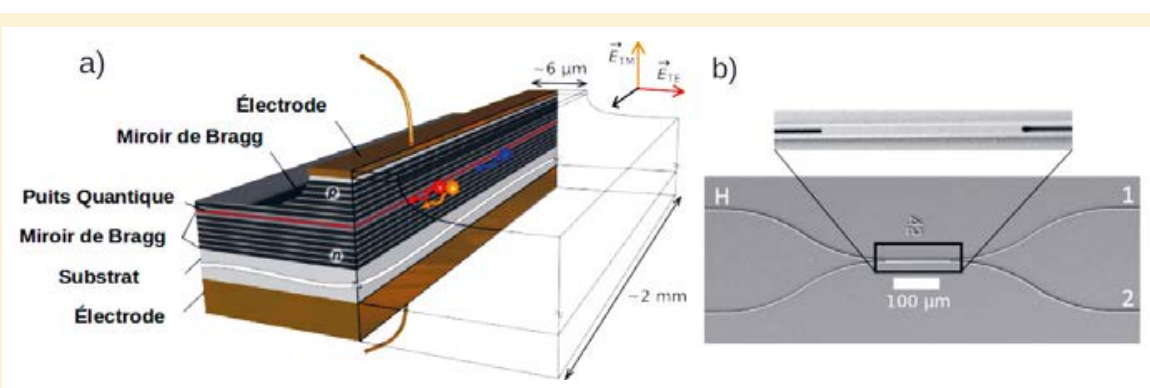

Figure 4. (a) Diode laser émettant des paires de photons : la structure, contenant un puits quantique, est optimisée pour générer l'émission laser et la convertir directement en paires de photons à l'intérieur du même dispositif. La condition d'accord de phase est obtenue par l'ingénierie de la dispersion des modes guidés par la structure, appelée accord de phase modal. (b) Intégration monolithique d'une source de photons uniques annoncés et d'un coupleur 50/50 : un faisceau laser de pompe en incidence verticale sur un des guides d'entrée génère des paires de photons se propageant dans des directions opposées. Un photon de chaque paire est détecté afin de signaler la présence du photon jumeau, ce qui réalise une source de photons uniques annoncés. La pureté quantique des photons émis est confirmée en analysant la statistique temporelle des photons transmis par les deux guides d'onde en sortie. (Crédits images : Laboratoire MPQ Université Paris Diderot/CNRS)

plage de transparence: sous certaines conditions, la réponse non-linéaire du matériau à un faisceau laser de pompe se traduit par l'émission de paires de photons corrélés (figure 2). Bien que dans ce cas la production de paires soit probabiliste, cette deuxième approche est la plus largement répandue car elle est simple à mettre en ouvre et permet de faire l'ingénierie d'une grande variété d'états quantiques à température ambiante et dans la bande spectrale des télécommunications.

\section{Sources intégrées de photons intiviqués}

\section{Les boîtes quantiques}

Les boites quantiques sont des nanostructures semi-conductrices qui confinent les électrons et les trous dans les trois dimensions de l'espace ; leurs niveaux d'énergie sont alors quantifiés comme ceux d'un atome, si bien qu'elles sont parfois appelées «atomes artificiels ». Sous excitation optique ou électrique, une boîte quantique peut capturer deux électrons et deux trous pour former un état bi-excitonique (état lié électron-trou). Un des électrons se recombine avec un des trous en émettant un premier photon; la paire électron-trou restante se recombine ensuite en générant un deuxième photon-on parle de cascade bi-excitonique (figure 1). Pour exploiter ce processus il est nécessaire que l'énergie thermique du système soit inférieure à l'énergie des états excitoniques, ce qui impose un refroidissement cryogénique des dispositifs. Les systèmes à base de boîtes quantiques ont des brillances record pour l'émission de photons uniques et présentent aussi un intérêt particulier en tant qu'interface entre deux types de qubits : le photon et le spin associé aux porteurs de charge.

\section{Les guides d'onde en cristaux diélectriques}

Les cristaux non-linéaires diélectriques sont les systèmes les plus largement utilisés aujourd'hui (et disponibles commercialement) pour produire des paires de photons par mélange à trois ondes; parmi eux, le niobate de lithium $\left(\mathrm{LiNbO}_{3}\right.$ ou LN) permet de fabriquer des guides d'onde avec un taux de production de paires élevé en utilisant la technique de quasi-accord-de-phase (figure 3a), ainsi que des modulateurs électro-optiques très rapides. Malgré la taille macroscopique de ces systèmes (plusieurs centimètres) et leur incompatibilité avec la technologie CMOS, plusieurs démonstrations de puces intégrant les sources non-linéaires et différents éléments optiques linéaires ont déjà été réalisées et des travaux sont également en cours pour utiliser ces systèmes en régime de variables continues (comme les quadratures du champ électrique) [3].

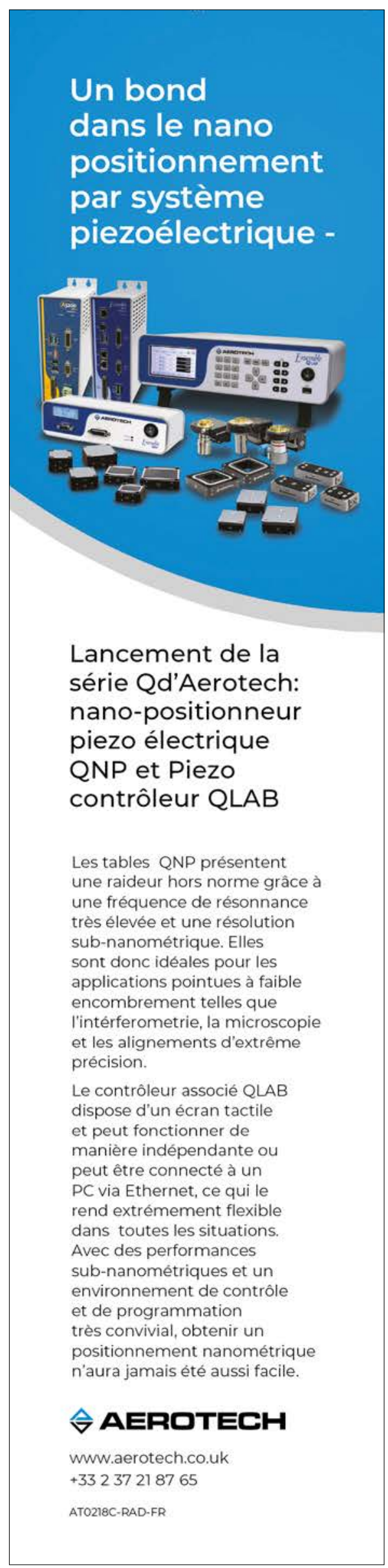




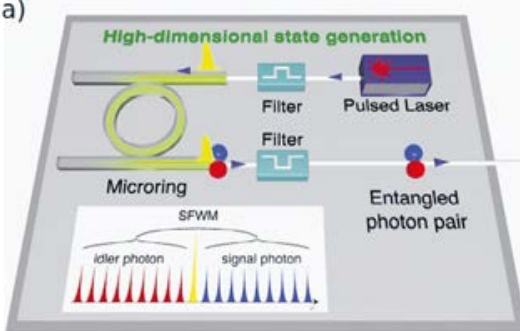

b)

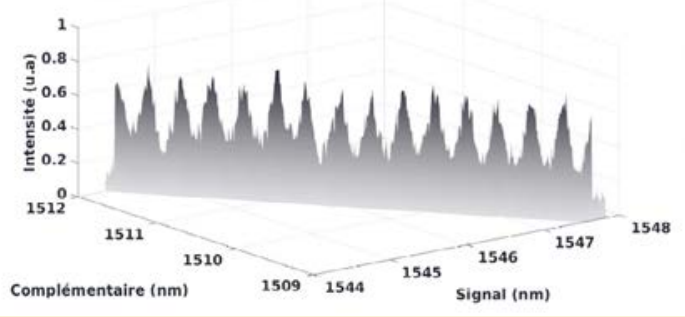

\section{Les plateformes silicium, SiN et Hydex}

Les développements récents de la photonique silicium sur isolant (SOI) ont permis de démontrer des sources de photons intriqués par mélange à quatre ondes en utilisant différents types de géométries : guides d'onde ruban, spirales, résonateurs micro-anneau (figure $3 b$ )... Cette plateforme est particulièrement intéressante pour sa compatibilité avec la technologie CMOS, utilisée dans la fabrication des circuits électroniques intégrés, et pour la possibilité d'intégrer les sources d'états quantiques avec un grand nombre de composants déjà existants pour le routage, la commutation et le traitement de signaux photoniques. Ces dernières années, pour contourner la limitation liée à l'absorption à deux photons de la plateforme SOI aux longueurs d'onde télécom, des progrès remarquables ont été effectués sur deux autres plateformes compatibles CMOS: le nitrure de silicium $(\mathrm{SiN})$ et la silice dopée à fort indice (Hydex) (figure 5a) [4] .

\section{La plateforme AlGaAs}

Les semiconducteurs à gap direct constituent également une plateforme de choix pour la photonique quantique intégrée. En particulier, le mélange à trois ondes dans des guides d'onde en AlGaAs permet de générer des paires de photons intriqués tout en gardant la possibilité d'intégrer le laser de pompe
Figure 5. Génération de qudits intriqués en fréquence avec un résonateur non-linéaire à micro-anneau en Hydex (a) (crédit image Michael Kues UOP Group, NRS-EMT Montreal, Canada) et un guide d'onde AlGaAs (b) (crédit image Laboratoire MPQ, Université Paris Diderot/ CNRS). Dans les deux cas les photons jumeaux sont générés à des longueurs d'onde symétriques par rapport à la dégénérescence, et dans une superposition quantique des modes en fréquence définis par les résonances de la cavité. effet électro-optique de l'AlGaAs pour contrôler la phase et réaliser des interférences quantiques sur puce. Les guides d'onde AlGaAs offrentégalement un terrain de choix pour la génération d'états quantiques de lumière en exploitant des variables continues. En particulier, la grande largeur spectrale offerte par l'accord de phase modal permet de générer des états fortement intriqués en fréquence. Combiné aux effets de cavitéliés à la réflectivité des facettes du guide, ceci permet de générer des états où les corrélations prennent la forme d'un peigne de fréquences (figure 5b). Les photons peuvent constituer alors des qudits, des unités d'information pouvant prendred valeurs distinctes, où $d \sim 300$ correspond au nombre de dents du peigne. Ceci constituerait un gain de deux ordres de grandeur dans la densité d'information par rapport à l'utilisation de la polarisation du photon (où $d=2$ correspond aux deux polarisations orthogonales). injectée électriquement et fonctionnant à température ambiante (figure 4a) [5]. Par ailleurs, la maturité technologique de la plateforme AlGaAs permet d'ores et déjà de réaliser des circuits photoniques quantiques combinant sur une même puce la génération et la manipulation d'états quantiques de lumière. La figure $4 b$ montre l'exemple d'un circuit AlGaAs formé d'un coupleur 50/50 comportant deux guides d'onde en entrée et en sortie; ce dispositif a permis de montrer pour la première fois sur cette plateforme la génération et l'analyse de photons uniques annoncés [6].

Cette démonstration ouvre la voie à la réalisation de circuits plus complexes, utilisant notamment le fort

\section{Conclusion}

Les sources intégrées de photons intriqués sont en train de jouer un rôle de tout premier plan dans le développement des futures technologies quantiques. Un grand nombre de dispositifs basés sur divers types de matériaux sont en cours de développement et très certainement des solutions hybrides verront le jour pour combiner les avantages de chaque plateforme et générer, manipuler et détecter des nouveaux états de la lumière sur puce. Des recherches passionnantes s'annoncent tant pour les théoriciens que pour les expérimentateurs!

\section{POUR EN SAVOIR PLUS}

[1] A. Einstein, B. Podolski, N. Rosen, Can quantum mechanical description of physical reality be considered complete? Phys. Rev. 47, 777 (1935)

[2] A. Orieux et al., Semiconductor devices for entangled photon pair generation: a review, Rep. Prog. Phys. 80, 076001 (2017)

[3] O. Alibart et al., Quantum photonics at telecom wavelengths based on lithium niobate waveguides, J. Opt. 18, 104001 (2016)

[4] D.J. Moss et al., New CMOS-compatible platforms based on silicon nitride and Hydex for nonlinear optics, Nat. Photon. 7, 597 (2013)

[5] F. Boitier et al., Electrically Injected Photon-Pair Source at Room Temperature, Phys. Rev. Lett. 112, 183901 (2014)

[6] J. Belhassen et al., On-chip III-V monolithic integration of heralded single photon sources and beamsplitters, Appl. Phys. Lett. 112, 071105 (2018) 